\title{
AUXIN RESPONSE FACTOR 6 (ARFG) and ARF8 promote Gibberellin-mediated hypocotyl xylem expansion and cambium homeostasis
}

\author{
Mehdi Ben-Targem ${ }^{1}$, Dagmar Ripper ${ }^{1}$, Martin Bayer ${ }^{2}$ and Laura Ragni ${ }^{1 *}$ \\ ${ }^{1}$ ZMBP- Center for Plant Molecular Biology, University of Tübingen, Auf der Morgenstelle 32, \\ D-72076 Tübingen, Germany, ${ }^{2}$ Max Planck Institute for Developmental Biology, Max-Planck- \\ Ring 5 , 72076 Tübingen, Germany \\ *Corresponding author: laura.ragni@zmbp.uni-tuebingen.de +49 (0)7071 - 2976677
}

Mehdi Ben-Targem: mehdibt86@gmail.com

Dagmar Ripper: dagmar.ripper@zmbp.uni-tuebingen.de

Martin Bayer: 0000-0001-5806-2253; martin.bayer@tuebingen.mpg.de

Laura Ragni: 0000-0002-3651-8966; laura.ragni@zmbp.uni-tuebingen.de

\section{Keywords:}

Xylem, Phloem, Cambium, Auxin responsive factor, Arabidopsis hypocotyl 


\begin{abstract}
During secondary growth, the thickening of plant organs, wood (xylem) and bast (phloem) are continuously produced by the vascular cambium. In Arabidopsis hypocotyl and root, we can distinguish two phases of secondary growth based on cell morphology and production rate. The first phase, in which xylem and phloem are equally produced, precedes the xylem expansion phase in which xylem formation is enhanced and xylem fibers differentiate. It is known that Gibberellins (GA) trigger this developmental transition via the degradation of DELLA proteins and that the cambium master regulator BREVIPEDICELLUS/KNAT1 (BP/KNAT1) and the receptor like kinases ERECTA and ERL1 regulate this process downstream of GA. However, our understandings on the regulatory network underlying GA-mediated secondary growth, are still limited.
\end{abstract}

Here, we demonstrate that DELLA-mediated xylem expansion is mainly achieved through RGA and GAI and that RGA and GAI promote cambium senescence. We further show that AUXIN RESPONSE FACTOR (ARF6) and ARF8, which physically interact with DELLAs, specifically repress phloem proliferation and induce cambium senescence during the xylem expansion phase. Moreover, the inactivation of $B P$ in arf6 arf8 background revealed an essential role for ARF6 and ARF8 in cambium establishment and maintenance. Overall, our results shed light on a pivotal hormone cross-talk between GA and auxin in the context of plant secondary growth.

\title{
Introduction
}

Secondary growth, the increase in girth of plant organs, largely contributed to the success of land plants. Secondary growth is mainly driven by the vascular cambium, a post-embryonic meristem, which divides in a strictly bifacial manner, producing xylem (wood) inward and phloem outward (Ragni \& Greb, 2018). In perennial dicotyledons, xylem tissue represents the principal form of biomass accumulation (Demura \& Ye, 2010; Spicer \& Groover, 2010). In addition, the vasculature contributes to the transport of assimilate, ions, water and signaling molecules and confers mechanical strength. Several studies suggest that the Arabidopsis hypocotyl is a valid model to study secondary growth and wood production (Chaffey et al., 2002; Ragni \& Hardtke, 2014), as it has been shown that key regulators are conserved between herbaceous and woody plants (Barra-Jimenez \& Ragni, 2017) and extensive amounts of secondary xylem fibers and vessel 
elements are produced in the hypocotyl, with structural and ultra-structural characteristics similar to those of trees (Chaffey et al., 2002).

In the Arabidopsis hypocotyl, secondary growth is characterized by two distinct growth phases. Upon termination of elongation at around 2-3 day-after germination, the hypocotyl grows only radially, xylem and phloem are produced at the same rate and the xylem comprises only vessels (first phase). In the second growth phase or the so called "xylem expansion" phase, xylem differentiates at higher rate than phloem and xylary fibers are formed (Sibout et al., 2008). This developmental transition in the hypocotyl is induced by flowering in many herbaceous annual plants with rosette habitus including Arabidopsis (Ragni et al., 2011). However, neither bolting nor flower specification per se are necessary. (Ragni et al., 2011). Grafting experiments indicated the presence of a mobile signal, which at flowering is translocated from the shoot to the hypocotyl to induce xylem expansion (Sibout et al., 2008). The phytohormone Gibberellin (GA) acts as the mobile cue that is transported at flowering to the hypocotyl where it drives DELLA degradation and promotes the xylem expansion program (Ragni et al., 2011). Indeed, xylem expansion is enhanced in della quadruple mutants, in which GA signaling is constitutively turned on and abolished by the local expression of a non-degradable version of DELLA (Ragni et al., 2011).

DELLA proteins are conserved components of the GA signaling pathway acting immediately downstream of the GA receptor, regulating a plethora of developmental processes and responses to abiotic stresses (Locascio et al., 2013; Colebrook et al., 2014; Davière \& Achard, 2016). They control gene expression by sequestering transcription factors and repressors or by participating in transcriptional complexes (Locascio et al., 2013). Consistently, they have been shown to interact with several transcription factor families (Marin-de la Rosa et al., 2014) including: MYBs, NACs, and the AUXIN RESPONSIVE FACTORs /ARFs. For instance, RGA sequesters ARFs activators (ARF6, ARF7 and ARF8), but not the repressor ARF1 to regulate hypocotyl elongation (Oh et al., 2014). Recently, it has been shown that RGA binds also to the transcription factor BREVIPEDICELLUS/KNAT1 (BP/KNAT1), a known secondary growth regulator (Liebsch et al., 2014; Woerlen et al., 2017; Felipo-Benavent et al., 2018). Inactivation of BP leads to impaired cambial activity and absence of fiber differentiation. In addition, $b p$ mutant do not form hypocotyl fibers even upon GA application indicating that BP acts also during the xylem expansion phase (Ikematsu et al., 2017). Additionally ERECTA (ER) and ERECTA-LIKE1 (ERL1), two receptor like kinases, which are involved in many developmental processes such as 
stomata patterning and stem elongation (Shpak, 2013) regulate the transition to the xylem expansion in a BP-dependent manner (Ikematsu et al., 2017).

The extent of xylem expansion varies among different natural accessions. In a pioneer study Sibout et al. identified several natural accessions with contrasting phenotypes (Sibout et al., 2008), whereas we reported that the common laboratory strains Columbia (Col) and Landsberg erecta (Ler) greatly differ in the magnitude of xylem expansion. Ler has enhanced xylem expansion compared to Col, while Col displayed larger overall secondary growth than Ler (Ragni et al., 2011; Sankar et al., 2014). Surprisingly, the difference in xylem expansion among the two accessions cannot be explained by the inactivation of ERECTA, which occurs in the Ler ecotype (Ikematsu et al., 2017). In a recent study, genome-wide association studies (GWAS) unraveled the role of another receptor like kinase SUPPRESSOR OF BRI1 /EVERSHED (SOBIR/EVR) during secondary growth (Milhinhos et al., 2019). SOBIR, which acts together with ERECTA, prevents precocious fiber formation. Moreover, SOBIR expression is negatively regulated by BP (Milhinhos et al., 2019). Nevertheless the GA signaling network underlying secondary growth is still poorly understood and so far BP/KNAT1 is the only known protein physically interacting with DELLA (Felipo-Benavent et al., 2018), which regulates xylem expansion (Liebsch et al., 2014; Woerlen et al., 2017; Felipo-Benavent et al., 2018).

In this work, we show that RGA and GAI are the main members of the DELLA family regulating the transition to the xylem expansion phase, highlighting their role in modulating cambium senescence (decrease of cambium activity at the last plant developmental phase). We expanded the GA downstream network by integrating ARF6 and ARF8 as two DELLA-interacting proteins and we demonstrated that ARF6 and ARF8 repress phloem proliferation and regulate cambium activity. Interestingly, arf6 arf8 double mutants show ectopic phloem proliferation only after the onset of xylem expansion phase and delayed cambium senescence, highlighting their specific function in this developmental transition. In addition, the inactivation of BP in arf6 af8 background results in a nearly total arrest of secondary growth, unmasking a possible ARF6 and ARF8 function in establishing and maintaining active vascular cambium.

\section{Experimental procedure}

\section{Plant material and growth}


The majority of the lines used are in Col background unless otherwise specified in the text/figure. All plants used for confocal microscopy are in Col background, grown in vitro (1/2 MS with $0.1 \%$ of sugar) in continuous light. For all the other experiments, plants were grown in soil in long day conditions (16 hours light versus 8 hours dark) and the sampling time is stated in the text/figure. nph4-1, arf6-2, arf8-3, arf6-2 nph4-1 arf8-3/+, pRPS5a::GAL4 and pUAS::MIR167 and the F1 issued from the cross between them were kindly provided by Jason Reed (University of North Carolina, USA) and described in (Nagpal et al., 2005) (Stowe-Evans et al., 1998). arf8-7 (Gutierrez et al., 2009) ARF6:GUS and ARF8:GUS (Nagpal et al., 2005; Wu et al., 2006) were kindly provided by Catherine Bellini (Umeå university, Sweden). The double rga-24 gai-t6 was a gift from Markus Schmid (Umeå university, Sweden). The dellako, er-105 and SUC2:GUS were previously described in (Feng et al., 2008) (Torii et al., 1996) and (Schulze et al., 2003). rga-28, gai-tdl and the double rga-28 gai-tdl (Plackett et al., 2014) were provided by Stephen Thomas (Rothamsed Research). bp-9 (Pautot et al., 2001), BP:GUS (Pautot et al., 2001) are gift from V.Pautot (INRA Versailles), RGA:rgaD-GR, GAI:gaiD-GR, rga-24, gai-t6 were previously described in (Ragni et al., 2011) ARF6::NLS-3xGFP (N67078), ARF7::NLS-3xGFP (N67080), ARF8::NLS-3xGFP (N67082) arf6-1(N24606) and arf8-2(N24608) (Okushima et al., 2005) were ordered from NASC. RGA:rgaD-GR, GAI:gaiD-GR in Col-0 background were obtained by backcrossing 6 times the original lines to Col-0. arf6-1 arf8-2/+, bp-9 arf6-2 arf8-3/+, BP:GUS arf6-2 arf8-3/+, arf6-2 rga-28 gai-td1/+ arf8-3/+ were generated by crossing and genotyping. Primers for genotyping are listed in Table S1.

\section{GA and Dexamethasone Treatment}

Induction of soil grown $R G A: r a^{D}-G R$, or $G A I: g a i^{D}-G R$ plants in Ler or Col-0 background was achieved by watering with a $10 \mu \mathrm{M}$ dexamethasone solution three times per week until hypocotyl sampling. Plants grown invitro, were treated by submerging in a liquid $10 \mu \mathrm{M}$ dexamethasone (dex) 1/2 Murashige and Skoog solution for 3 hours upon flowering. For GA and

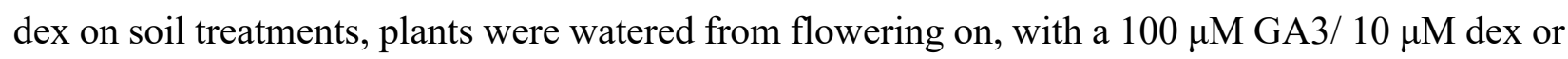
mock solution at similar frequency three times per week until hypocotyl sampling according to the experiment.

\section{Molecular Cloning}


The RGA promoter was amplified with the primers: A-pRGA F (AACAGGTCTCAACCTTATAACCTCATCCATCTATAG) and Br-pRGA R (AACAGGTCTCATGTTTCAGTACGCCGCCGTCGAGAG). The BsaI site inside the pRGA promoter was removed by overlapping PCRs and cloned in pGG-A0. The GAI promoter was $\begin{array}{lllll}\text { amplified } & \text { with } & \text { the } & \text { primers: }\end{array}$ (AACAGGTCTCAACCTTGGGACCACAGTCTAAATGGCGT) and Br-pGAI R (AACAGGTCTCATGTTGGTTGGTTTTTTTTCAGAGATGGA) and cloned in pGG-A0. The promoters were assembled in pZ03 with the available published modules to obtain RGA:NLSGFP-GUS and GAI: NLS-GFP-GUS by Green Gate technologies (Lampropoulos et al., 2013).

\section{Histology and stainings}

Thin plastic cross-sections were obtained from plastic embedded material using TECHNOVIT 7100 (Heraeus Kulzer) and a Leica RT microtome as described in (Barbier de Reuille \& Ragni, 2017). GUS essay was performed according to the protocol described in (Wunderling et al., 2018). Vibratome sections $(50-80 \mu \mathrm{m})$ were obtained via a Leica VT-1000 vibratome, from hypocotyls embedded in 6\% agarose block, slices were collected in water in microscope slides, stained and/or imaged. For Phloroglucinol staining, a ready solution (VWR, 26337.180) was applied directly to the section. Images were taken with a Zeiss Axio M2 imager microscope. For sections of fluorescent lines the ClearSee protocol described in (Ursache et al., 2018) was applied. Briefly, the hypocotyls were first fixed in a 4\% PFA solution with $0.01 \%$ Triton for 1 hour. and then embedded in 5\% Agarose for vibratome cutting. Vibratome sections are then directly collected in 1x PBS solution. After incubation, the 1x PBS solution is removed and replaced by ClearSee solution. The sections are subsequently kept at room temperature in ClearSee solution for at least 1 to 2 days. Finally, the samples are incubated for $20 \mathrm{~min}$ at RT in 0,05\% Calcofluor White then washed and mounted on slides in ClearSee solution (Kurihara et al., 2015).

\section{Images acquisition}

A Zeiss Axio M2 imager microscope or a Zeiss Axiophot microscope was used to take images at different magnifications of vibratome sections $(50-80 \mu \mathrm{m})$ as well as $5 \mu \mathrm{m}$ sections stained with $0.1 \%$ toluidine blue solution. Pictures of vibratome sections of fluorescent lines were acquired using the Zeiss LSM880 confocal microscope. GFP ex. 488 em. 490-520. Calcofluor White ex 404 em: 430-450. 


\section{Image analyses and statistical analyses}

The total hypocotyl cross section area, the xylem area and the fiber area were analyzed using ImageJ software as previously described (Sibout et al., 2008; Wunderling et al., 2017). Statistical analyses were performed using IBM SPSS Statistics version 24-25 (IBM). We first tested all datasets for homogeneity of variances using Levene's Test of Equality of Variances. For multiple comparison, we calculated the significant differences between each dataset using One way ANOVA with Tamhane's post hoc (equal variance not assumed) or a Bonferroni correction (equal variance assumed). The significance threshold was set to $\mathrm{p}$-value $<0.05$. For comparing two groups, we used Welch's t-test (not homogenous variance) or a Student's t-test (homogeneous variance), $\mathrm{P}$ values are indicated in the figure legends.

\section{Results}

\section{GA signaling controls hypocotyl secondary growth, mostly through RGA and GAI, in Ler and Col-0 ecotypes.}

We previously showed that della quadruple mutants are characterized by an increased xylem expansion and fiber production (Ragni et al., 2011), however, the specific contribution of each DELLA during secondary growth, is unknown. Thus, we analyzed della single knock-out mutants. Only rga mutants exhibited a mild but significant increase of xylem to total area ratio (from now on referred as xylem occupancy) in comparison to wildtype (Figure S1), suggesting a certain degree of redundancy. As $G A I$ has been shown to play overlapping roles with $R G A$ in several developmental processes (Dill \& Sun, 2001; Cheng et al., 2004), we investigated the rga24 gai-t6 double mutants. rga-24 gai-t6 mutants showed a strong increase in xylem occupancy and fiber formation (Figure S1c-d), whereas knocking out all DELLAs (dellako) only slightly enhanced the rga gai phenotype, suggesting that $R G A$ and $G A I$ are the major regulators of xylem expansion (Figures S1c-d).

Plakett el al. showed that rga gai double mutants are sterile in Col background, whereas they set seeds in Ler background (Plackett et al., 2014). As Ler and Col also greatly differ in secondary growth morphodynamics with Col displaying more overall secondary growth and Ler more xylem expansion (Ragni et al., 2011; Sankar et al., 2014), we investigated the vascular phenotype of rga gai in both ecotypes. Both rga-24 gai-t6 (Ler background) and rga-28 gai-td1 
(Col background) displayed enhanced xylem expansion, compared to their wild-type (WT) counterparts, however, the absolute values reflect the differences between the two ecotypes with rga-24 gai-t6 having 50\% xylem occupancy (in Ler) and rga-28 gai-tdl only 30\% (in Col) (Figures 1a-b). Interestingly, the triple mutant rga-28 gai-tdl er-105 was undistinguishable from the rga28 gai-tdl double mutant in terms of xylem expansion (Figures 1a-b) pointing out that the difference between the two double mutants is not due to the inactivation of ERECTA in Ler background. Consistently, it was previously shown that, the loss of function of ERECTA (in Col background) does not increase xylem expansion (Ikematsu et al., 2017) (Figures 1a-b). To corroborate our results, we studied the effect of the induction of a non-degradable version of RGA and GAI (GAI:gaiD17-GR and RGA:rgaD17-GR), in both backgrounds. To this extent, the original GAI:gaiD17-GR and RGA:rgaD17-GR Ler lines (Ragni et al., 2011) have been backcrossed 6 times to Col background. Induction, upon flowering, of a DELLA version that cannot be degraded in the presence of GA resulted in reduced xylem occupancy and fiber formation in both accessions (Figures S2a-b). Altogether these results suggest that in Ler background a xylem promoting or phloem repressing factor acts in a DELLA- and ERECTA-independent pathway to promote the xylem expansion phase. Thus, to easily characterize the GA downstream pathway, we mainly exploited the Col background.

In Col ecotype, we observed further secondary growth phenotypes when we perturbed GA signaling possibly due to the absence of "the xylem promoting factor". Induction of gaiD17 or rgaD17 upon flowering, promotes ectopic cell divisions in the phloem and resulted in an increase in total hypocotyl area (Figures 1c and S2c). Consistently, rga-28 gai-td1 mutants (Col background) are characterized by a reduction in total hypocotyl area (Figure 1d). This led us to further investigate cambium activity in the context of xylem expansion. For this purpose, we measured the number of cambial derivative layers (CD layers), at the onset (5 daf), during early and late xylem expansion (15-30 daf). We observed that in WT hypocotyls during the late xylem expansion phase, the number of CD layers decrease, ultimately leading to cambium consumption at plant senescence, from now on this phenomenon will be referred to as cambium senescence (Figure $\mathrm{S} 2 \mathrm{~d}$ ). By contrast, the number of cambium cell layers in dex-treated RGA:rgaD-GR plants was increased compared to mock (20 daf) indicating that DELLAs delay cambium senescence (Figures 1e-f). This is in line with the fact that rga-28 gai-tdl double mutants displayed less cambial layers than WT (10 daf) (Figures S2e-f), indicating that DELLA proteins delay cambium 
consumption during senescence.

\section{BP is not the only factor required for fiber formation:}

BP has been shown to regulate fiber differentiation during xylem expansion through its physical interaction with DELLA (Felipo-Benavent et al., 2018) bp mutants (in Col ecotype) lack fiber differentiation and are insensitive to GA (Ikematsu et al., 2017). This is in contrast to what we observed in $b p$ mutants in Ler ecotype $(b p-1)$, which can still partially respond to GA application (Figures S3a-b). bp-1 mutant plants were able to form fibers albeit at lower extent and only very late in development (20 daf), suggesting that fiber formation is possible in the absence of BP, in a background in which xylem expansion is enhanced (Figure S3a).

To better clarify this issue, we investigated whether the inactivation of RGA and GAI rescues the $b p$ phenotype (in Col background). In rga gai bp-9, plant height phenotype was partially rescued (Figure S3c) and we observed fiber formation at the time of plant senescence, nevertheless, the altered xylem occupancy was not rescued by the inactivation of RGA and GAI. Altogether these results indicate that $\mathrm{BP}$ is not the only factor required for the xylem expansion phase (Figures S3d-f).

\section{ARF6, ARF7 and ARF8 expression patterns overlap with RGA and GAI during hypocotyl secondary growth.}

In the quest for GA downstream factors controlling hypocotyl secondary growth, we thought that ARF6, ARF7 and ARF8 are promising candidates, as they physically interact with RGA and GAI (Oh et al., 2014) and auxin is a key regulator of vascular patterning and xylem differentiation (Ragni \& Greb, 2018). To investigate whether ARF6, ARF7 and ARF8 regulate the xylem expansion phase through the interaction with DELLA, we first checked whether their expression patterns overlap with RGA and GAI during secondary growth. RGA and GAI are broadly expressed in the hypocotyl before flowering (Figure S4a), whereas at flowering time, $R G A$ and $G A I$ expression gets progressively restricted to the phloem poles. During xylem expansion, RGA and GAI are still expressed at the phloem poles albeit at lower levels (10 day-after-flowering). Consistently RGA protein accumulation was reported in the phloem (Felipo-Benavent et al., 
2018)(Figure 2a, b). Interestingly, $A R F 6, A R F 7$ and $A R F 8$ expression was also detected in the phloem throughout secondary growth, $A R F 6$ and $A R F 7$ expression was broader and encompassed the cambium and differentiating xylem respectively, similar to their expression pattern in the root, described by (Smetana et al., 2019) (Figures 2c-e). In addition, ARF6 and ARF7 and to a lesser extent ARF8 expression was maintained also after flowering. To conclude, RGA, GAI, ARF6, ARF7 and $A R F 8$ expression patterns overlap in the phloem, therefore fulfilling the requirement that these factors could physically interact also during secondary growth.

\section{ARF6, ARF8 and to a lesser extent ARF7 promote xylem expansion, repressing phloem proliferation.}

To further investigate the ARF-DELLA genetic interaction during secondary growth, we tested whether arf mutants displayed altered xylem expansion. arf7/nph4 mutants are characterized by reduced total hypocotyl area but did not show any xylem expansion phenotype (Ragni et al., 2011). arf6 (arf6-1 and arf6-2) and arf8 (arf8-2 and arf8-3) single mutants showed a slight but significant inhibition of xylem expansion and less fibers compared to the WT background (Figs. 3 a-d and S4c). Whereas the $\operatorname{arf6} \operatorname{arf8}$ (arf6-2 $\operatorname{arf8-3}$ and $\operatorname{arf6}-1$ arf8-2) double mutants displayed a dramatic decrease in xylem occupancy and absence of fiber accumulation until very late stages of plant growth (30-40 days after flowering in our growth conditions) (Figures 3a-d and S4c). Interestingly in arf6 arf8 double mutants, we observed the presence of phloem fibers at plant senescence, which rarely occur in WT plants (Figure 3b), an increased number of phloem poles and ectopic cell divisions at phloem poles (Figure 3e and S4d) reminiscent of the phenotypic consequences of impaired GA signaling in Col background (Figure 1c). To confirm that this phloem phenotype is due to the inactivation of $A R F 6$ and $A R F 8$, we took advantage of the fact that the expression of $A R F 6$ and $A R F 8$ is tightly regulated by miR167a (Wu et al., 2006; Gutierrez et al., 2009). Supporting the role of $A R F 6$ and $A R F 8$, we observed ectopic cell divisions in the phloem of F1 plants, in which we ectopically expressed miR167a (UAS::miR167ax RPS5a::GAL4) (Figure $\mathrm{S} 4 \mathrm{e})$. These results suggest that the transition to the xylem expansion phase relies on reducing cambium cell differentiation into phloem, resulting in a shift towards xylem differentiation.

We next wondered about the temporal dynamics of ARF6 and ARF8 DELLA interaction: if RGA sequesters ARFs during the first phase of secondary growth, then we would expect that 
arf6 arf8 mutants show strong changes in secondary growth only after flowering. Consistently with this hypothesis, arf6 arf8 hypocotyls were undistinguishable from WT plants at flowering time (arf6 arf8 double mutants flower at the same day as WT in long day conditions) (Figures S4f). After flowering (from $\cong 10$ daf), however, xylem occupancy starts to increase in wild-type plants, whereas in $\operatorname{arf6} \operatorname{arf} 8$ (at 15 daf) it was unchanged and only increased mildly at 30 daf (Figures S5ab). By contrast, we remarked that total hypocotyl area was bigger in arf6 arf8 double mutants compared to WT (Figures S5c). This could be explained by prolonged cambium activity, in arf6 arf8 mutants. At the onset of xylem expansion and during early xylem expansion phase (5-15daf),

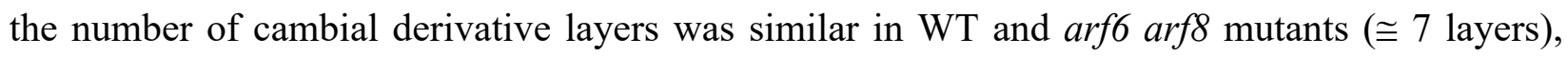
whereas in the late xylem expansion phase (at 30 daf), cambial activity was decreased in WT but not in arf6 arf 8 plants ( $\cong 4$ layers vs $\cong 6$ layers), indicating that $A R F 6$ and $A R F 8$ promote cambium consumption (Figures S5d, e).

Finally, we wondered whether the inactivation of ARF7 could enhance the arf6 arf 8 phenotype. arf7/nph4 arf6 arf8 displayed a further reduction of xylem occupancy (Figure S5f), compared to the double mutant, however the ectopic phloem cell divisions phenotype was not enhanced (Figure 3f and S5g), suggesting only a minor contribution by $A R F 7$.

\section{ARF6, ARF7 and ARF8 act mainly downstream of GA signaling to control xylem expansion.}

To further confirm the interaction between DELLA and ARFs and its significance in controlling GA mediated xylem expansion, we tested whether GA responses are attenuated in arf6 arf8 double mutants. This was achieved by exogenous GA application and by inactivating $R G A$ in arf6 arf8 background. As previously shown, GA treatment enhances fiber production and increases xylem occupancy in WT (Ragni et al., 2011) (Figures 4a-c), by contrast arf6 arf8 mutants were only partially able to respond to GA as indicated by the mild increase in xylem occupancy and fiber production, (Figure 4a-c). Moreover, in arf7/nph4 arf6 arf8 triple mutants, GA treatment, did not increase xylem occupancy and fiber accumulation was abolished in the majority of the plants (Figures 4a-c). In line with this observation, rga ar6 arf8 triple mutants showed decreased xylem occupancy and no fiber production compared to rga single mutant (Figures S6a-c). Similarly, arf6 arf8 rga gai/+ showed less xylem occupancy and fibers compared to rga gai/+ (Figures S6a-c). Over all, these results confirm that $A R F 6, A R F 7$ and $A R F 8$ are required to mediate GA dependent 
xylem expansion. The partial restoration of the arf6 arf8 phenotype caused by DELLA inactivation or GA treatment could be explained by the additional role of ARF7 in xylem expansion or ectopic BP activity in arf6 arf8 mutants (see below).

\section{BP inactivation completely abolishes xylem expansion and secondary growth in arf6 arf8}

To investigate the interaction and the specific contribution of BP and ARF6 ARF8 during xylem expansion, we generated arf6 arf8 $b p$ triple mutants. At 25daf, arf6 arf8 $b p$ triple mutant displayed a dramatic reduction of xylem occupancy when compared to arf6 arf8 double or $b p$ mutants (Figures 5a-b), indicating that they act in independent pathways. Moreover, loss of ARF6 and $A R F 8$ in $b p$ mutants (arf6 arf8 $b p$ ) leads to a drastic reduction of total hypocotyl area/overall secondary growth compared to $b p$ single mutants (Figures 5a-c), suggesting that ARF6 and ARF8 regulate cambium activity during all phases of secondary growth. To further clarify this, we investigated cambium development in $\operatorname{arf6}$ arf8 $b p$ triple and $\operatorname{arf6} b p$ and $\operatorname{arf} 8 \mathrm{bp}$ double mutant combinations. At flowering time, both double and triple mutants already displayed reduced xylem area and number of cambial layers when compared to $b p$ (Figures 5d-e and S6d), demonstrating that ARF6 and ARF8 promote cambial activity in the absence of BP. Strikingly, cambium development was perturbed to such a degree in arf6 arf8 $\mathrm{bp}$ mutants that it was not possible to distinguish the typical cambial cell morphology (Figure 5d). Consistently only few secondary xylem vessels were produced (Figure 5f). Altogether these results indicate that ARF6 and ARF8 genetically interact with BP to promote cambium establishment and activity during the early phase of secondary growth.

\section{Discussion:}

Secondary growth results in the thickening of plant organs and formation of secondary xylem (wood), which is a principal sink for carbon and a fundamental source of natural renewable energy (Demura \& Ye, 2010). It largely relies on the interdependent processes of cell proliferation and differentiation (Ragni \& Greb, 2018). GA positively controls secondary growth by modulating cambial activity and fiber differentiation (Eriksson et al., 2000; Björklund et al., 2007; Ragni et al., 2011; Ikematsu et al., 2017). Differences in GA signaling define two separate phases of secondary growth in Arabidopsis hypocotyl; a first phase in which xylem and phloem are produced 
at the same rate and a second phase (the so-called xylem expansion) in which xylem production is accelerated and xylem fiber differentiates (Ragni et al., 2011). The transition between these two phases is triggered by local GA-dependent degradation of DELLA proteins, which occurs in the hypocotyl at flowering time (Ragni et al., 2011).

In this study, we further elucidated the GA-mediated DELLA modulation of secondary growth. In Arabidopsis, the DELLA gene family comprises 5 members (Zentella et al., 2007) and analyses of della single and higher order mutants pointed out that $R G A$ and $G A I$ are the major contributors. The role of $R G A$ and $G A I$ in secondary growth is conserved across ecotypes, which greatly differ in xylem expansion dynamics such as Col and Ler. GA signaling, through the degradation of RGA and GAI, triggers xylem expansion and cambium senescence in both accessions and this is independent of ERECTA, a known regulator of xylem expansion, which is not functional in Ler background. Interestingly, in both accessions, the expression of undegradable versions of DELLA represses xylem occupancy but only in Col background promotes phloem development and prevents cambium senescence, revealing a new aspect of GA-mediated xylem expansion. This could be explained by the fact that in Ler accession an unknown factor possibly promotes xylem formation independently of DELLA and ERECTA and the resultant high xylem occupancy masks the effect of GA on the cambium /phloem. In line with this idea, a similar scenario has been proposed in the context of flower fertility where an unidentified stamen elongation regulator, which is not ERECTA, promotes stamen elongation independently of DELLA in Ler background (Plackett et al., 2014).

The presence of a xylem promoting factor in Ler accession may also explain why the inactivation of BP in Ler background has a weaker fiber phenotype compared to the Col allele. Despite the major role of BP in promoting fiber formation during xylem expansion, our genetic analyses indicate that BP is not the only DELLA-interacting factor controlling fiber formation. Consistently, DELLA proteins are known to physically interact with many transcription factors belonging to different families (Claeys et al., 2014; Colebrook et al., 2014; Marin-de la Rosa et al., 2014) and act as central hub connecting several signaling cascades such as auxin, cytokinins, ethylene, Jasmonate and ABA in different developmental contexts (Locascio et al., 2013). It is well established that auxin promotes different aspects of secondary growth such as cambium establishment, cambium homeostasis, xylem differentiation and periderm formation (Brackmann et al., 2018; Smetana et al., 2019; Xiao et al., 2020). In this work we show that auxin and GA, via the interaction of ARF6, ARF8 with DELLA, regulate xylem expansion, revealing a novel 
hormonal cross-talk during secondary growth. ARF6 and ARF8 are known to regulate flower development, adventitious root formation, and hypocotyl elongation (Nagpal et al., 2005; Goetz et al., 2006; Gutierrez et al., 2012; Liu et al., 2014) and recently, we show that ARF8 promotes periderm growth in the root (Xiao et al., 2020). Consistently with their novel role during xylem expansion, we showed that $\operatorname{arf6} \operatorname{arf} 8$ mutants display reduced xylem occupancy, diminished xylary fiber formation, retarded cambium senescence, and enhanced phloem proliferation, mimicking the expression of a undegradable version of DELLA (in Col background). Moreover, GA responses are attenuated in arf6 arf 8 double mutants, and nearly abolished in arf6 arf7 arf 8 triple mutants validating the specific role played by DELLA-ARFs interaction in controlling xylem expansion. In addition, arf6 arf8 double mutants form phloem fibers, which hardly occurs in WT plants, highlighting the role of ARF6 and ARF8 in repressing phloem development. The presence of phloem fibers is in concordance with recent reports, showing that Jasmonate promotes phloem fiber formation (Behr et al., 2018), as it is known that $A R F 6$ and $A R F 8$ activate JA conjugating enzymes, limiting bioactive JA during adventitious root formation (Gutierrez et al 2012). During secondary growth, ARF6 and ARF8 are mainly expressed in the phloem, where also DELLA proteins accumulate, and in the cambium, indicating that the xylem phenotypes of arf6 arf 8 double mutants might be indirect effects of altered cambium/phloem function. These findings highlight, that xylem expansion does not only rely on promoting xylem and xylem fiber formation but also on the repression of phloem proliferation and promoting cambium senescence. To conclude, $A R F 6, A R F 7$, $A R F 8$ and $B P$ are sequestered and therefore inactivated by DELLA proteins before flowering, while after flowering ( the onset of xylem expansion) bioactive GAs accumulate in the hypocotyl (Talon et al., 1991; Ragni et al., 2011), triggering the degradation of DELLA proteins and the release ARFs and BP (Figure 6). Together ARFs and BP activate the transcriptional programs, which ultimately promotes xylem vessels and fiber production and repression of phloem proliferations.

Several ARFs such as ARF5/MONOPTEROS modulate cambium development. In the stem, ARF5 stimulates the transition of cambial stem cells into xylem cells by directly activating xylem-related genes and by repressing the key cambial regulator $W O X 4$, whereas ARF3 and ARF4 maintain cambial stem cell proliferation (Brackmann et al., 2018). By contrast, in the root ARF5 with ARF7 and ARF19, promotes the expression of class III HD-ZIPs, $P X Y$ and WOX4 triggering the establishment of the vascular cambium (Smetana et al., 2019). Similarly, in the hypocotyl at the onset of secondary growth ARF6 and ARF8 (in the absence of BP) promote cambium activity/ establishment probably by the activation of WOX4 as arf6 arf8 $b p$ triple mutants are reminiscent 
of wox4 bp double mutants (Zhang et al., 2019). Instead, in the late xylem expansion phase ARF6 and ARF8 activate cambial senescence. Consistently, the genetic interaction of $A R F 6, A R F 8$, and $B P$ varies among plant organs, in fact during flower development, $A R F 6$ and $A R F 8$ repress $B P$ expression and the inactivation of BP partially restores the arf6 arf8 flower defects (Tabata et al., 2010), whereas in the hypocotyl knocking out BP enhanced the xylem occupancy phenotype of arf6 arf8. This is in accordance with other studies showing that BP has different functions in above and underground (root/hypocotyl) organs (Mele et al., 2003; Liebsch et al., 2014; Woerlen et al., 2017).

Interestingly, both BP and ARFs interact with DELLA and are expressed in the phloem highlighting the possibility of higher order protein complex formation. Recently, Campbell et al showed that abscisic acid specifically stimulates fiber formation during xylem expansion independently of BP (Campbell et al., 2018). As DELLAs are known to bind ABA signaling core components such as ABSICS ACID INSENSITIVE 3 and ABI5 (ABI5) (Lim et al., 2013), it is tempting to speculate that the ABA-mediated fiber promotion is regulated by DELLA (Figure 6). Further research focusing on the spatio-temporal dynamics of DELLA, BP, ARFs, and ABI protein accumulation and interaction, coupled with tissue-specific target analyses will help to further dissect the distinct events that lead to xylem expansion, and contrasting functions of ARFs and BP during all stages of secondary growth.

\section{Conflict of Interest}

The authors declare no conflict of interest.

\section{Authors contribution}

LR, MBT, and MB designed and LR and MBT conducted the experiments, MBT performed the histology with the help of DR. LR, DR and MBT generated the plant lines. LR and MBT wrote the manuscript with the help of MB.

\section{Funding}

LR is indebted to the Baden-Württemberg Stiftung for financial support of this research project by the Elite program for Postdocs. This project was also supported by the seed funding of the SFB1101.

\section{Acknowledgement:}


We thank Stefan Mahn for cloning the DELLA promoters and Andrea Boch for helping with plant cultivation. We thank Ari Pekka Mähönen for discussion and reading of the manuscript.

\section{Supporting Information}

Fig. S1 RGA and GAI are the main DELLA regulating secondary growth.

Fig. S2 Induction of rgaD17 and gaiD17 repress xylem expansion in both Col and Ler ecotypes.

Fig. S3 Genetic interaction between BP and DELLA.

Fig. S4 DELLA and ARF expression pattern and arfs mutant characterization.

Fig. S5 Secondary growth dynamics in $\operatorname{arf6} \operatorname{arf} 8$ double mutants.

Fig. S6 Genetic interaction between ARFs and DELLA.

Table S1 Primers used for genotyping.

\section{Figure legends:}

\section{Fig. 1 GA signalling controls hypocotyl secondary growth, mostly through $R G A$ and $G A I$, in} Ler and Col ecotypes.

(a) Plastic cross-sections of 10 day-after-flowering (daf) hypocotyls showing xylem expansion in WT (Ler and Col background), rga-24-gai-t6 (in Ler background), rga-28 gai-td1 (in Col background), er-105 (in Col background) and rga-28 gai-td1 er-105 (in Col background). Doubleheaded red arrows indicate the xylem. Black scale bars:100 $\mu \mathrm{m}$ (b) Quantification of Xylem Area/Total area of the experiment showed in (a). Letters in the graphs refer to individual groups in a one-way ANOVA analysis with a post-hoc multiple group T-test (n=11-19). (c) Plastic crosssections of 15daf hypocotyls of rga:rgaD17-GR and gai:gaiD17-GR in Col. Plants were treated with $10 \mu \mathrm{M}$ DEX from flowering until sample collection. Blue arrows indicate ectopic cell divisions in the phloem. Black scale bar: $100 \mu \mathrm{m}$. (d) Quantification of Total Area of the experiment showed in (a). Letters in the graphs refer to individual groups in a one-way ANOVA analysis with a posthoc multiple group T-test $(\mathrm{n}=11-19)$. (e) Magnification of (c) showing the cambium of rga:rgaD17-GR mock and treated hypocotyls. Double-headed orange arrows indicate the cambium. Black scale bar: $20 \mu \mathrm{m}$. (f) Quantification of the number of cambium derivative layers (CD layers) of the experiment showed in (e). T-test $(\mathrm{n}=6, * * *: \mathrm{p}<0.001)$. 
Fig. $2 A R F 6$ and $A R F 8$ expression pattern overlaps with $R G A$ and $G A I$ during hypocotyl secondary growth.

(a-d) Hypocotyl vibratome cross-sections at 0,8 and 20 day-after-flowering. Left and middle panels: confocal images of sections cleared with ClearSee and stained with Calcofluor-White showing GFP signal in the nuclei (red arrows). Right panels: GUS assay on vibratome sections stained with Phloroglucinol (red arrows indicates the phloem). (a) RGA:NLS-GFP-GUS (left and middle panels) and RGA:GUS (right panel). (b) GAI:NLS-GFP-GUS (left and middle panels)and GAI:GUS (right panel). (c) ARF6:NLS-3xGFP (left and middle panels) and ARF6:GUS (right panel). (d) ARF8:NLS-3xGFP (left and middle panels) and ARF8:GUS(right panel). (a-d) White scale bar: $20 \mu \mathrm{m}$ and black scale bars: $20 \mu \mathrm{m}$.

Fig. 3 ARF6, ARF8 and to a lesser extent ARF7 promotes xylem expansion, repressing phloem proliferation.

(a) Plastic hypocotyl cross-sections of 10 day-after-flowering (daf), showing reduced xylem expansion in arf6, arf8 and arf6 arf8 mutants compared to WT (Col). (b) Phloroglucinol stained vibratome sections of 40 daf hypocotyl, showing reduced fibers formation in arf6, arf8 and arf6 arf8 mutants compared to WT (Col). (c) Quantification of the Xylem Area/Total area ratio of the experiment showed in (a). Letters in the graphs refer to individual groups in a one-way ANOVA analysis with a post-hoc multiple group T-test (n=9-17). (d) Quantification of Fiber Area/Xylem Area ratio of the experiment showed in (b). Letters in the graphs refer to individual groups in a one-way ANOVA analysis with a post-hoc multiple group T-test ( $n=2-18)$. (e) Magnifications of (a) showing the phloem region. (f) Plastic hypocotyl cross-sections of 10 daf, showing reduced xylem expansion and ectopic cell divisions in the phloem in arf6, arf8 double and arf6 nph4/arf7arf8 triple mutants compared to WT. Black scale bars: $100 \mu \mathrm{m}$. Double-headed red arrows indicate the xylem, double-headed black arrows indicate xylem fibers, black arrow indicate phloem fibers and blue arrows indicate ectopic divisions in the phloem.

Fig. 4 ARF6, ARF7 and ARF8 act mainly downstream GA signalling to control xylem expansion.

(a) Plastic hypocotyl cross-sections of 10 day-after-flowering, showing xylem expansion in WT (Col), arf6 arf8 and arf6 nph4/arf7 arf8 plants treated with Mock or 10 $\mu \mathrm{M}$ GA solution. (b) 
Quantification of Xylem Area/Total area ratio of the experiment showed in (a). Letters in the graphs refer to individual groups in a one-way ANOVA analysis with a post-hoc multiple group T-test n=4-13). (c) Quantification of Fiber Area/Xylem Area ratio of the experiment showed in (a). Letters in the graphs refer to individual groups in a one-way ANOVA analysis with a post-hoc multiple group T-test $n=4-13$ ). Black scale bars: $100 \mu \mathrm{m}$. Double-headed red arrows indicate the xylem, double-headed black arrows indicate xylem fibers and blue arrows indicate ectopic divisions in the phloem.

\section{Fig. 5 BP inactivation completely abolishes secondary growth in arf6 arf8}

(a) Plastic hypocotyl cross-sections of 25 day-after-flowering (daf), showing xylem expansion in Col-0, bp, arf6 arf8 and ar6 arf8 bp mutants. Black scale bars: 100 $\mu \mathrm{m}$. (b) Quantification of the Xylem Area /Total area ratio of the experiment showed in (a). Letters in the graphs refer to individual groups in a one-way ANOVA analysis with a post-hoc multiple group T-test (n=12-23).

(c) Quantification of the Total area of the experiment showed in (a). Letters in the graphs refer to individual groups in a one-way ANOVA analysis with a post-hoc multiple group T-test (n=12-23).

(d) Plastic hypocotyl cross-sections of flowering Col-0, $b p$, arf6 $b p$, arf8 $b p$ and ar6 arf8 $b p$ mutants. Black scale bars: $50 \mu \mathrm{m}$. (e) Quantification of the number of cambium derivative layers (CD layers) of the experiment showed in (d). Letters in the graphs refer to individual groups in a one-way ANOVA analysis with a post-hoc multiple group T-test $(\mathrm{n}=4-7)$. T-test $(*: \mathrm{p}<0.05)$ and NQ (Not possible to quantify). (f) Quantification of the number of xylem vessels of the experiment showed in (d). Letters in the graphs refer to individual groups in a one-way ANOVA analysis with a post-hoc multiple group T-test $(n=4-7)$. Double-headed red arrows indicate the xylem, doubleheaded orange arrows indicate the cambium and green arrows indicate altered cambial morphology in arf6 arf8 $b p$ triple mutant.

\section{Fig. 6 arf6 arf8 Model explaining GA-mediated xylem expansion.}

In Arabidopsis hypocotyls, we can distinguish two phases of secondary growth. In the first phase xylem and phloem are produced at the same rate, whereas in the second phase, xylem production is enhanced and xylem fiber differentiated. Before flowering, GA levels are low and DELLA proteins sequester ARFs, BP and probably ABIs, blocking the activation of xylem expansion genes. At flowering, GA accumulates in the hypocotyl and mediates DELLA degradation, freeing ARFs, 
$\mathrm{BP}$ and probably ABIs, which promote cambium senescence, phloem repression and fiber production.

\section{References:}

Barbier de Reuille P, Ragni L. 2017. Vascular Morphodynamics During Secondary Growth. Methods Mol. Biol. 1544: 103-125.

Barra-Jimenez A, Ragni L. 2017. Secondary development in the stem: when Arabidopsis and trees are closer than it seems. Current Opinion in Plant Biology 35: 145-151.

Behr M, Lutts S, Hausman JF, Guerriero G. 2018. Jasmonic acid to boost secondary growth in hemp hypocotyl. Planta 248(4): 1029-1036.

Björklund S, Antti H, Uddestrand I, Moritz T, Sundberg B. 2007. Cross-talk between gibberellin and auxin in development of Populus wood: gibberellin stimulates polar auxin transport and has a common transcriptome with auxin. Plant J 52(3): 499-511.

Brackmann K, Qi J, Gebert M, Jouannet V, Schlamp T, Grünwald K, Wallner E-S, Novikova DD, Levitsky VG, Agustí J, et al. 2018. Spatial specificity of auxin responses coordinates wood formation. Nat. Commun. 9(1): 875.

Campbell L, Etchells JP, Cooper M, Kumar M, Turner SR. 2018. An essential role for abscisic acid in the regulation of xylem fibre differentiation. Development 145(21): dev161992.

Chaffey N, Cholewa E, Regan S, Sundberg B. 2002. Secondary xylem development in Arabidopsis: a model for wood formation. Physiologia Plantarum 114(4): 594-600.

Cheng H, Qin L, Lee S, Fu X, Richards DE, Cao D, Luo D, Harberd NP, Peng J. 2004. Gibberellin regulates Arabidopsis floral development via suppression of DELLA protein function. Development 131(5): 1055-1064.

Claeys H, De Bodt S, Inze D. 2014. Gibberellins and DELLAs: central nodes in growth regulatory networks. Trends Plant Sci 19(4): 231-239.

Colebrook EH, Thomas SG, Phillips AL, Hedden P. 2014. The role of gibberellin signalling in plant responses to abiotic stress. J Exp Biol 217(Pt 1): 67-75.

Davière J-M, Achard P. 2016. A Pivotal Role of DELLAs in Regulating Multiple Hormone Signals. Molecular Plant 9(1): 10-20.

Demura T, Ye Z-H. 2010. Regulation of plant biomass production. Current Opinion in Plant Biology 13(3): 298-303.

Dill A, Sun T. 2001. Synergistic derepression of gibberellin signaling by removing RGA and GAI function in Arabidopsis thaliana. Genetics 159(2): 777-785.

Eriksson ME, Israelsson M, Olsson O, Moritz T. 2000. Increased gibberellin biosynthesis in transgenic trees promotes growth, biomass production and xylem fiber length. Nat Biotechnol 18(7): 784-788.

Felipo-Benavent A, Urbez C, Blanco-Tourinan N, Serrano-Mislata A, Baumberger N, Achard P, Agusti J, Blazquez MA, Alabadi D. 2018. Regulation of xylem fiber differentiation by gibberellins through DELLA-KNAT1 interaction. Development 145(23). 
Feng S, Martinez C, Gusmaroli G, Wang Y, Zhou J, Wang F, Chen L, Yu L, IglesiasPedraz JM, Kircher S, et al. 2008. Coordinated regulation of Arabidopsis thaliana development by light and gibberellins. Nature 451(7177): 475-479.

Goetz M, Vivian-Smith A, Johnson SD, Koltunow AM. 2006. AUXIN RESPONSE FACTOR8 is a negative regulator of fruit initiation in Arabidopsis. Plant Cell 18(8): 1873-1886.

Gutierrez L, Bussell JD, Pacurar DI, Schwambach J, Pacurar M, Bellini C. 2009. Phenotypic plasticity of adventitious rooting in Arabidopsis is controlled by complex regulation of AUXIN RESPONSE FACTOR transcripts and microRNA abundance. Plant Cell 21(10): 3119-3132.

Gutierrez L, Mongelard G, Flokova K, Pacurar DI, Novak O, Staswick P, Kowalczyk M, Pacurar M, Demailly H, Geiss G, et al. 2012. Auxin controls Arabidopsis adventitious root initiation by regulating jasmonic acid homeostasis. Plant Cell 24(6): 2515-2527.

Ikematsu S, Tasaka M, Torii KU, Uchida N. 2017. ERECTA-family receptor kinase genes redundantly prevent premature progression of secondary growth in the Arabidopsis hypocotyl. New Phytol 213(4): 1697-1709.

Kurihara D, Mizuta Y, Sato Y, Higashiyama T. 2015. ClearSee: a rapid optical clearing reagent for whole-plant fluorescence imaging. Development 142(23): 4168-4179.

Lampropoulos A, Sutikovic Z, Wenzl C, Maegele I, Lohmann JU, Forner J. 2013. GreenGate - A Novel, Versatile, and Efficient Cloning System for Plant Transgenesis. PLoS ONE 8(12): e83043.

Liebsch D, Sunaryo W, Holmlund M, Norberg M, Zhang J, Hall HC, Helizon H, Jin X, Helariutta Y, Nilsson O, et al. 2014. Class I KNOX transcription factors promote differentiation of cambial derivatives into xylem fibers in the Arabidopsis hypocotyl. Development 141(22): 4311-4319.

Lim S, Park J, Lee N, Jeong J, Toh S, Watanabe A, Kim J, Kang H, Kim DH, Kawakami N, et al. 2013. ABA-insensitive $3, A B A-i n s e n s i t i v e 5$, and DELLAs Interact to activate the expression of SOMNUS and other high-temperature-inducible genes in imbibed seeds in Arabidopsis. The Plant cell 25(12): 4863-4878.

Liu N, Wu S, Van Houten J, Wang Y, Ding B, Fei Z, Clarke TH, Reed JW, van der Knaap E. 2014. Down-regulation of AUXIN RESPONSE FACTORS 6 and 8 by microRNA 167 leads to floral development defects and female sterility in tomato. J Exp Bot 65(9): 25072520.

Locascio A, Blázquez MA, Alabadí D. 2013. Genomic Analysis of DELLA Protein Activity. Plant and Cell Physiology 54(8): 1229-1237.

Marin-de la Rosa N, Sotillo B, Miskolczi P, Gibbs DJ, Vicente J, Carbonero P, OnateSanchez L, Holdsworth MJ, Bhalerao R, Alabadi D, et al. 2014. Large-scale identification of gibberellin-related transcription factors defines group VII ETHYLENE RESPONSE FACTORS as functional DELLA partners. Plant Physiol 166(2): 1022-1032.

Mele G, Ori N, Sato Y, Hake S. 2003. The knotted1-like homeobox gene BREVIPEDICELLUS regulates cell differentiation by modulating metabolic pathways. Genes Dev 17(17): 20882093.

Milhinhos A, Vera-Sirera F, Blanco-Touriñán N, Mari-Carmona C, Carrió-Seguí À, Forment J, Champion C, Thamm A, Urbez C, Prescott H, et al. 2019. SOBIR1/EVR prevents precocious initiation of fiber differentiation during wood development through a mechanism involving BP and ERECTA. Proc Natl Acad Sci U S A 116(37): 1871018716. 
Nagpal P, Ellis CM, Weber H, Ploense SE, Barkawi LS, Guilfoyle TJ, Hagen G, Alonso JM, Cohen JD, Farmer EE, et al. 2005. Auxin response factors ARF6 and ARF8 promote jasmonic acid production and flower maturation. Development 132(18): 4107-4118.

Oh E, Zhu JY, Bai MY, Arenhart RA, Sun Y, Wang ZY. 2014. Cell elongation is regulated through a central circuit of interacting transcription factors in the Arabidopsis hypocotyl. Elife 3.

Okushima Y, Overvoorde PJ, Arima K, Alonso JM, Chan A, Chang C, Ecker JR, Hughes B, Lui A, Nguyen D, et al. 2005. Functional genomic analysis of the AUXIN RESPONSE FACTOR gene family members in Arabidopsis thaliana: unique and overlapping functions of ARF7 and ARF19. Plant Cell 17(2): 444-463.

Pautot V, Dockx J, Hamant O, Kronenberger J, Grandjean O, Jublot D, Traas J. 2001. KNAT2: evidence for a link between knotted-like genes and carpel development. Plant Cell 13(8): 1719-1734.

Plackett ARG, Ferguson AC, Powers SJ, Wanchoo-Kohli A, Phillips AL, Wilson ZA, Hedden P, Thomas SG. 2014. DELLA activity is required for successful pollen development in the Columbia ecotype of Arabidopsis. New Phytologist 201(3): 825-836.

Ragni L, Greb T. 2018. Secondary growth as a determinant of plant shape and form. Semin. Cell Dev. Biol. 79: 58-67.

Ragni L, Hardtke CS. 2014. Small but thick enough - the Arabidopsis hypocotyl as a model to study secondary growth. Physiologia Plantarum 151(2): 164-171.

Ragni L, Nieminen K, Pacheco-Villalobos D, Sibout R, Schwechheimer C, Hardtke CS. 2011. Mobile gibberellin directly stimulates Arabidopsis hypocotyl xylem expansion. Plant Cell 23(4): 1322-1336.

Sankar M, Nieminen K, Ragni L, Xenarios I, Hardtke CS. 2014. Automated quantitative histology reveals vascular morphodynamics during Arabidopsis hypocotyl secondary growth. Elife 3: e01567.

Schulze W, Reinders A, Ward J, Lalonde S, Frommer W. 2003. Interactions between coexpressed Arabidopsis sucrose transporters in the split-ubiquitin system.

Shpak ED. 2013. Diverse roles of ERECTA family genes in plant development. J Integr Plant Biol 55(12): 1238-1250.

Sibout R, Plantegenet S, Hardtke CS. 2008. Flowering as a condition for xylem expansion in Arabidopsis hypocotyl and root. Current Biology 18(6): 458-463.

Smetana O, Makila R, Lyu M, Amiryousefi A, Sanchez Rodriguez F, Wu MF, Sole-Gil A, Leal Gavarron M, Siligato R, Miyashima S, et al. 2019. High levels of auxin signalling define the stem-cell organizer of the vascular cambium. Nature 565(7740): 485-489.

Spicer R, Groover A. 2010. Evolution of development of vascular cambia and secondary growth. New Phytologist 186(3): 577-592.

Stowe-Evans EL, Harper RM, Motchoulski AV, Liscum E. 1998. NPH4, a Conditional Modulator of Auxin-Dependent Differential Growth Responses in Arabidopsis. Plant Physiology 118(4): 1265-1275.

Tabata R, Ikezaki M, Fujibe T, Aida M, Tian CE, Ueno Y, Yamamoto KT, Machida Y, Nakamura K, Ishiguro S. 2010. Arabidopsis auxin response factor6 and 8 regulate jasmonic acid biosynthesis and floral organ development via repression of class 1 KNOX genes. Plant Cell Physiol 51(1): 164-175.

Talon M, Zeevaart JA, Gage DA. 1991. Identification of Gibberellins in Spinach and Effects of Light and Darkness on their Levels. Plant Physiol 97(4): 1521-1526. 
Torii KU, Mitsukawa N, Oosumi T, Matsuura Y, Yokoyama R, Whittier RF, Komeda Y. 1996. The Arabidopsis ERECTA gene encodes a putative receptor protein kinase with extracellular leucine-rich repeats. Plant Cell 8(4): 735-746.

Ursache R, Andersen TG, Marhavy P, Geldner N. 2018. A protocol for combining fluorescent proteins with histological stains for diverse cell wall components. Plant J 93(2): 399-412.

Woerlen N, Allam G, Popescu A, Corrigan L, Pautot V, Hepworth SR. 2017. Repression of BLADE-ON-PETIOLE genes by KNOX homeodomain protein BREVIPEDICELLUS is essential for differentiation of secondary xylem in Arabidopsis root. Planta: 1-12.

Wu MF, Tian Q, Reed JW. 2006. Arabidopsis microRNA167 controls patterns of ARF6 and ARF8 expression, and regulates both female and male reproduction. Development 133(21): 4211-4218.

Wunderling A, Ben Targem M, Barbier de Reuille P, Ragni L. 2017. Novel tools for quantifying secondary growth. Journal of Experimental Botany 68(1): 89-95.

Wunderling A, Ripper D, Barra-Jimenez A, Mahn S, Sajak K, Targem MB, Ragni L. 2018. A molecular framework to study periderm formation in Arabidopsis. New Phytol 219: 216-229.

Xiao W, Molina D, Wunderling A, Ripper D, Vermeer JEM, Ragni L. 2020. Pluripotent Pericycle Cells Trigger Different Growth Outputs by Integrating Developmental Cues into Distinct Regulatory Modules. Curr Biol.

Zentella R, Zhang Z-L, Park M, Thomas SG, Endo A, Murase K, Fleet CM, Jikumaru Y, Nambara E, Kamiya Y, et al. 2007. Global Analysis of DELLA Direct Targets in Early Gibberellin Signaling in Arabidopsis. The Plant Cell Online 19(10): 3037-3057.

Zhang J, Eswaran G, Alonso-Serra J, Kucukoglu M, Xiang J, Yang W, Elo A, Nieminen K, Damén T, Joung JG, et al. 2019. Transcriptional regulatory framework for vascular cambium development in Arabidopsis roots. Nat Plants 5(10): 1033-1042. 
(a)

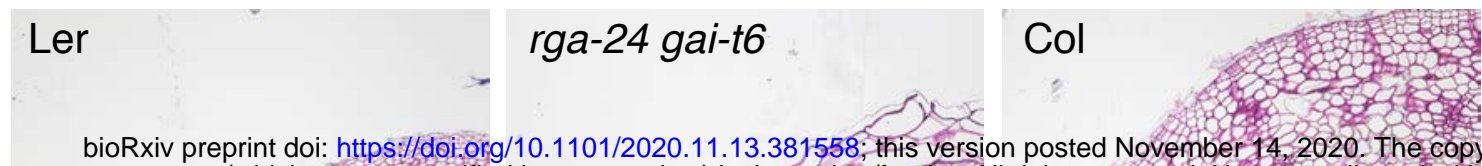

bioRxiv preprint doi: https:/doi.org/10.1101/2020.11.13.381558; this version posted November 14,2020. The copyright (which was not certified by peer review) is the author/funder. All rights reserved. No reuse allowed without $p$

(b)

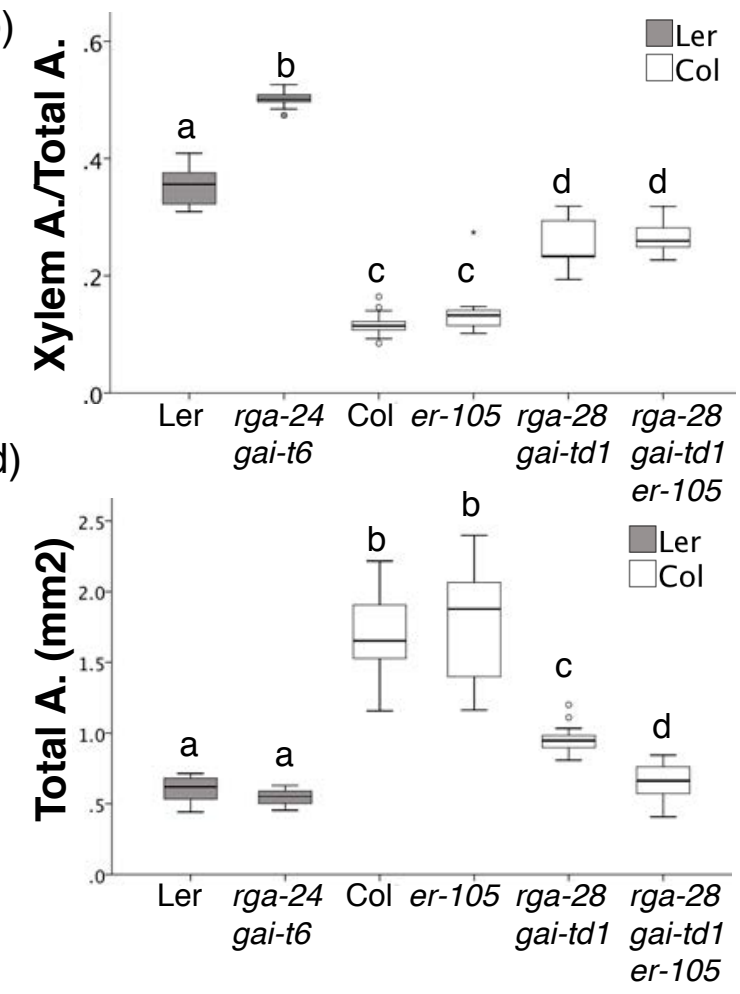

(e)

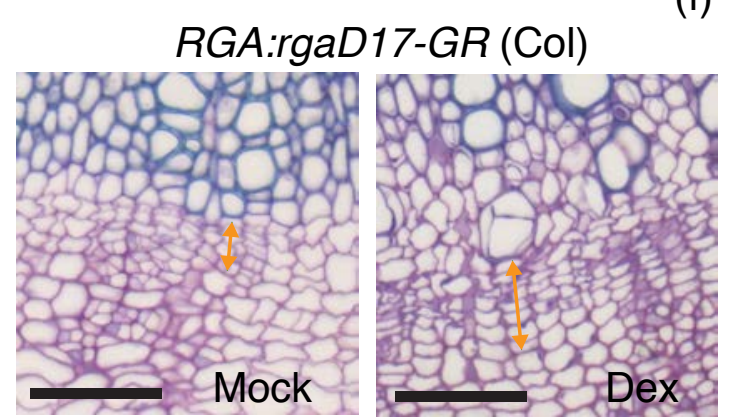

(d)

(f)

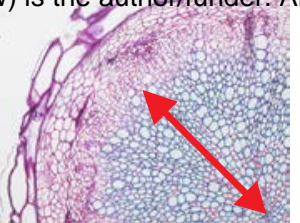

rga-28 gai-td1

er-105
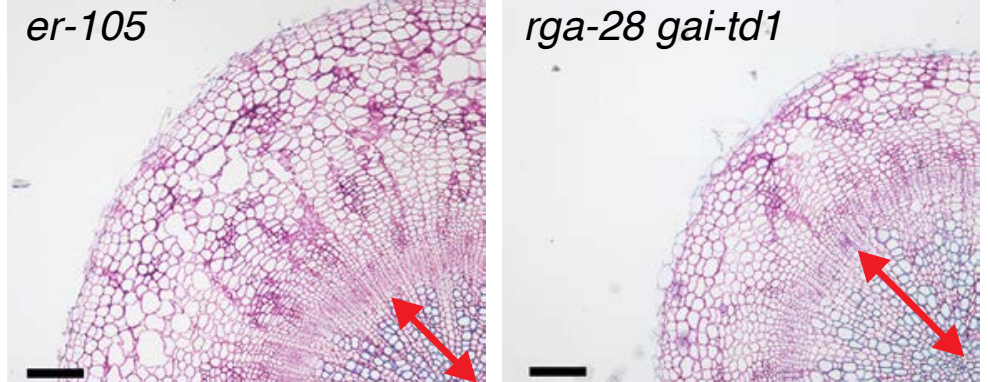

(c)

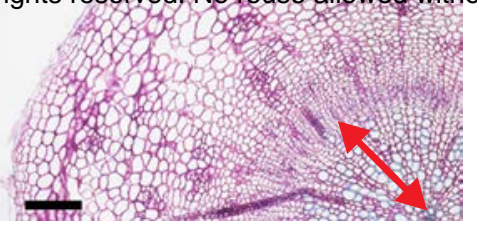

rga-28 gai-td1 er-105

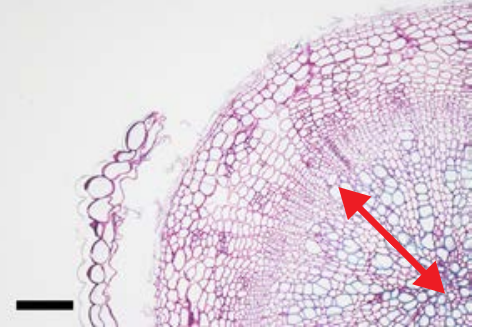

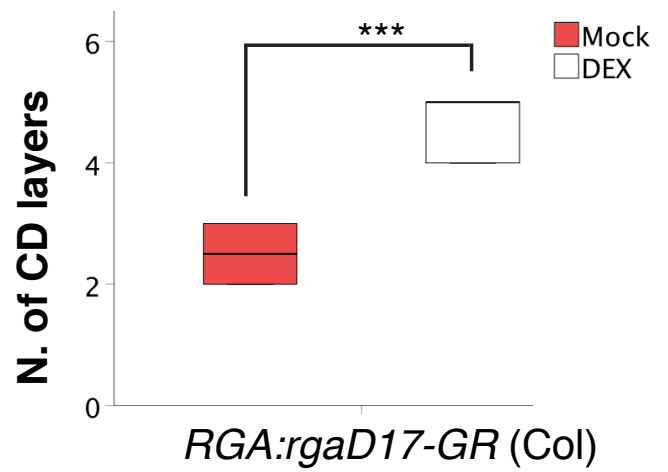

RGA:rgaD17-GR (Col)

Fig 1

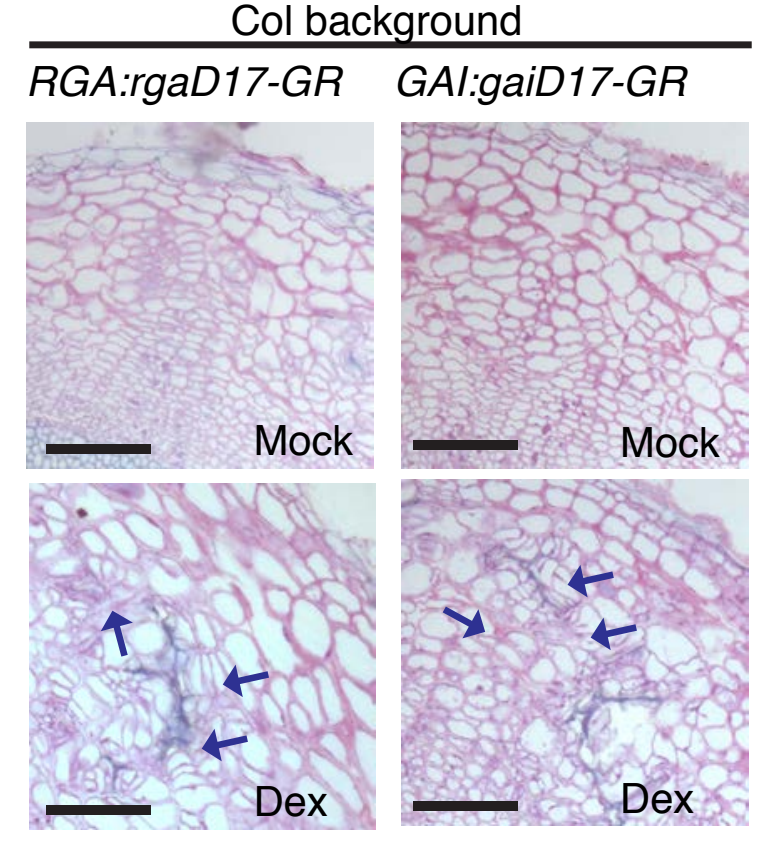


(a)

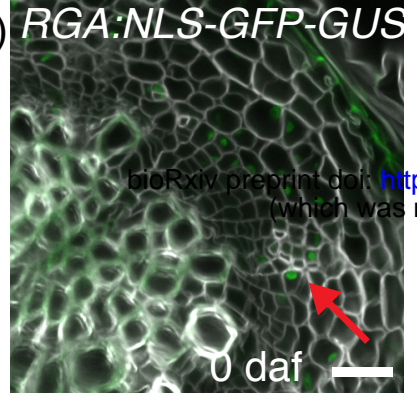

(b) GAI:NLS-GEP-GUS
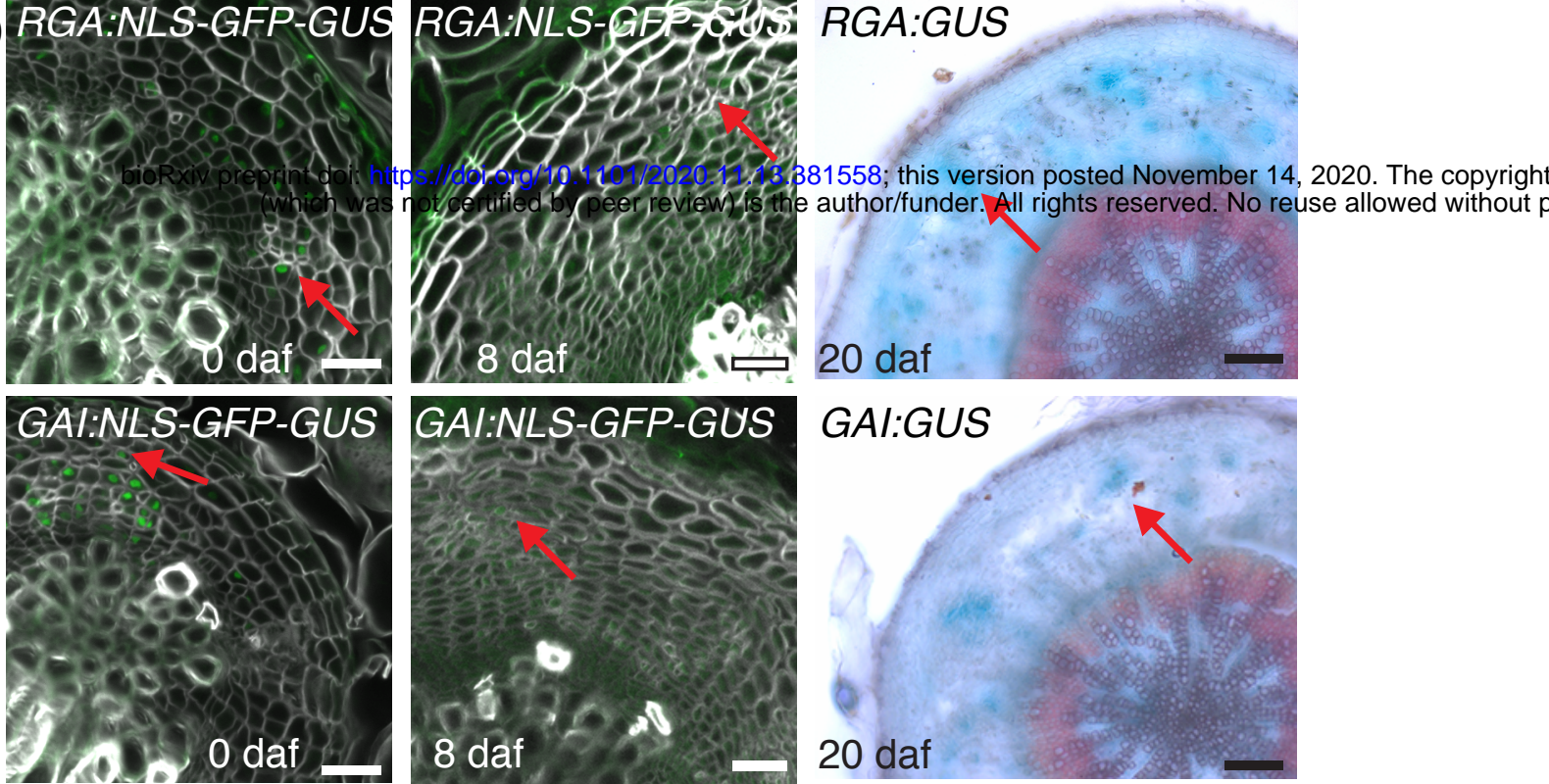

(c) ABE-ALSS-3XGEP
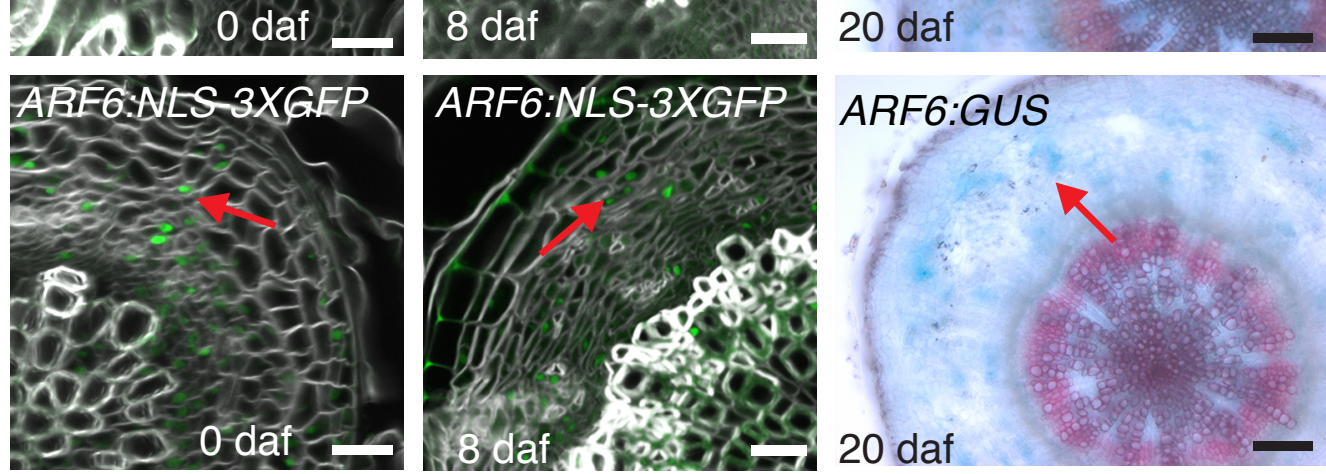

(d)
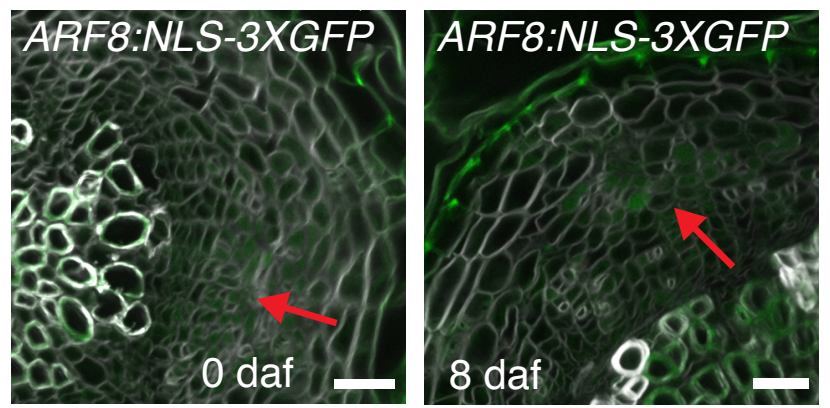

20 daf

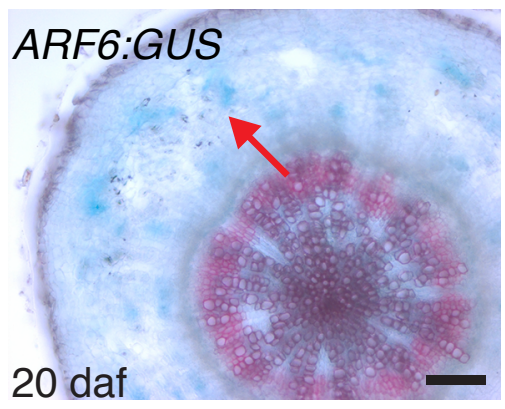

\section{ARF8:GUS}

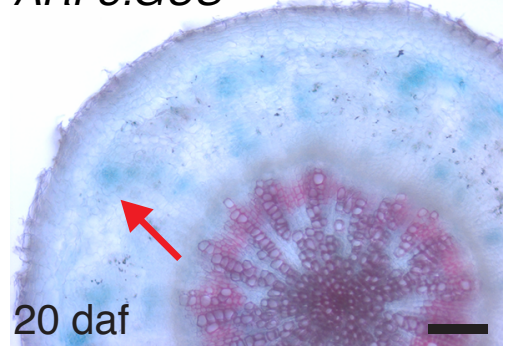

Fig. 2 
(a)

(b)

bioRxiv preprint doi: https:/doi.org/10 1 101/2020.11.13.381558; this version posted November 14,2020 . The copyright

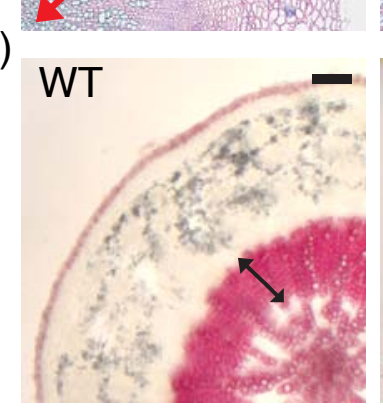

(c)
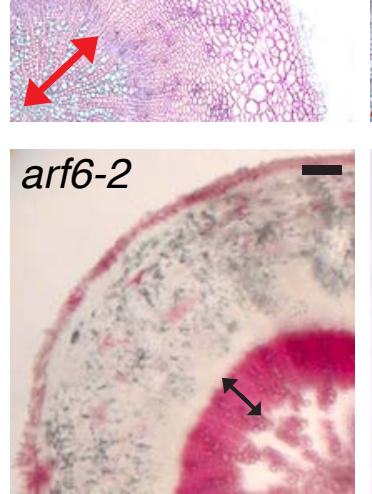

(d)
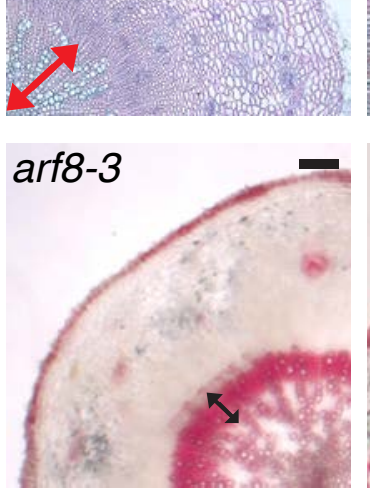

arfo- arfo-3

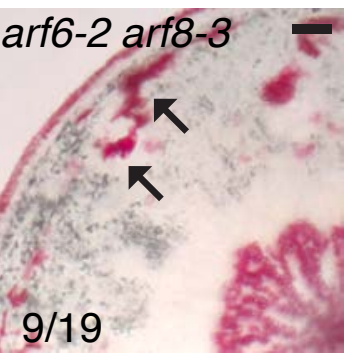

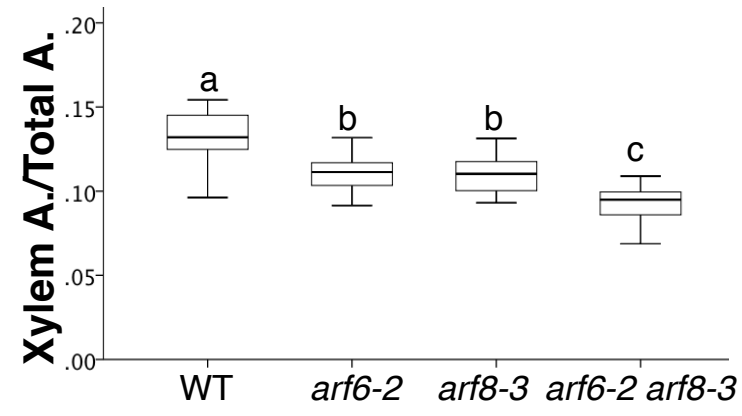

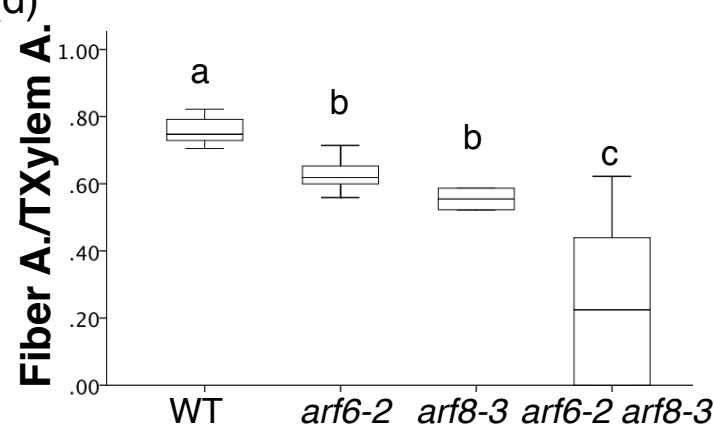

(e)

(f)
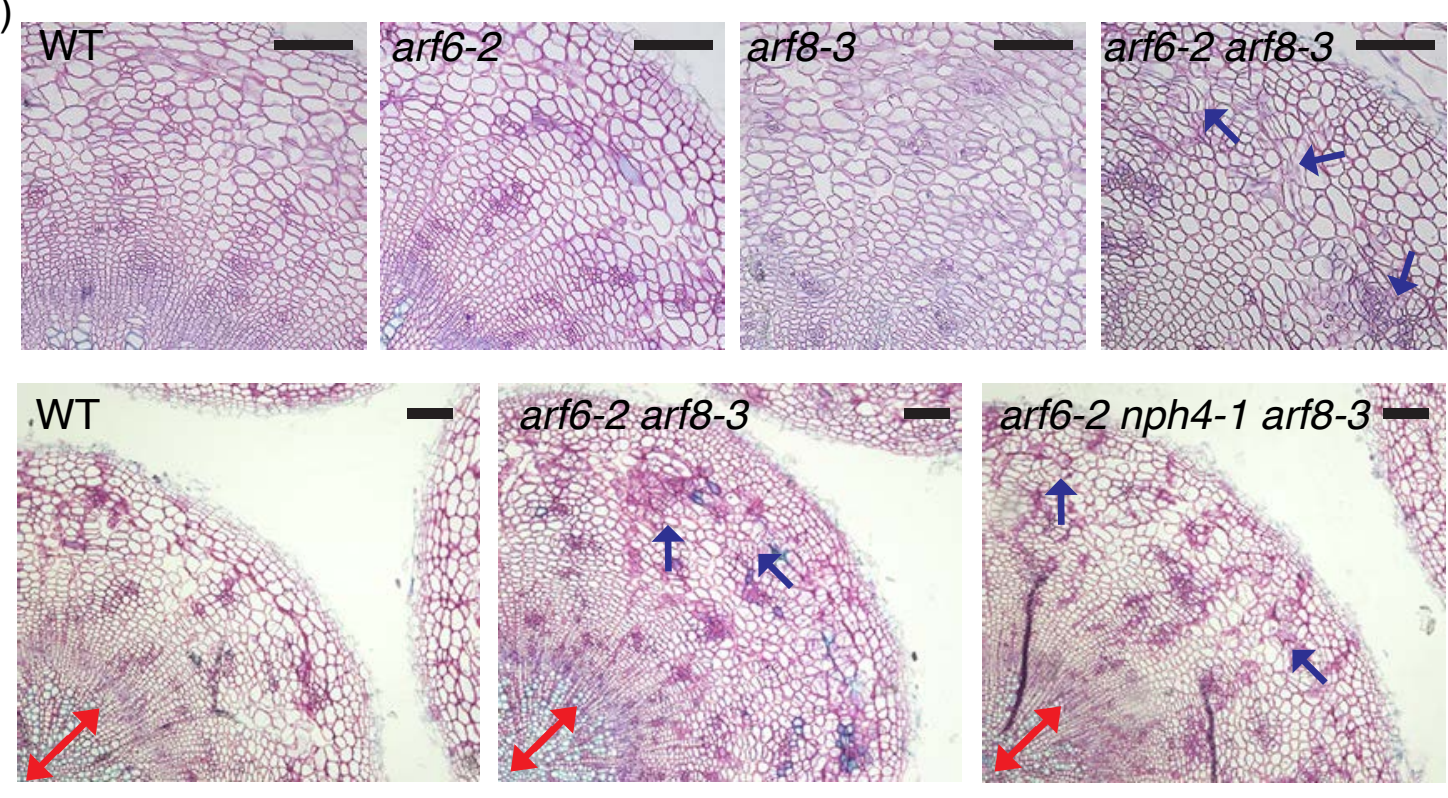

Fig.3 
(a)

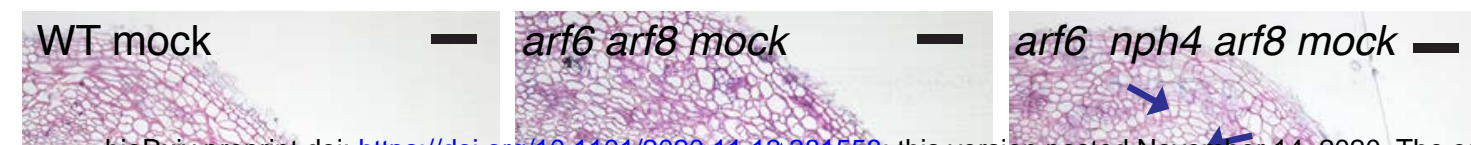

bioRxiv preprint doi: https://doi.org/10.1101/2020.11.131381558; this version posted Noventioer 14, 2020. The copyright
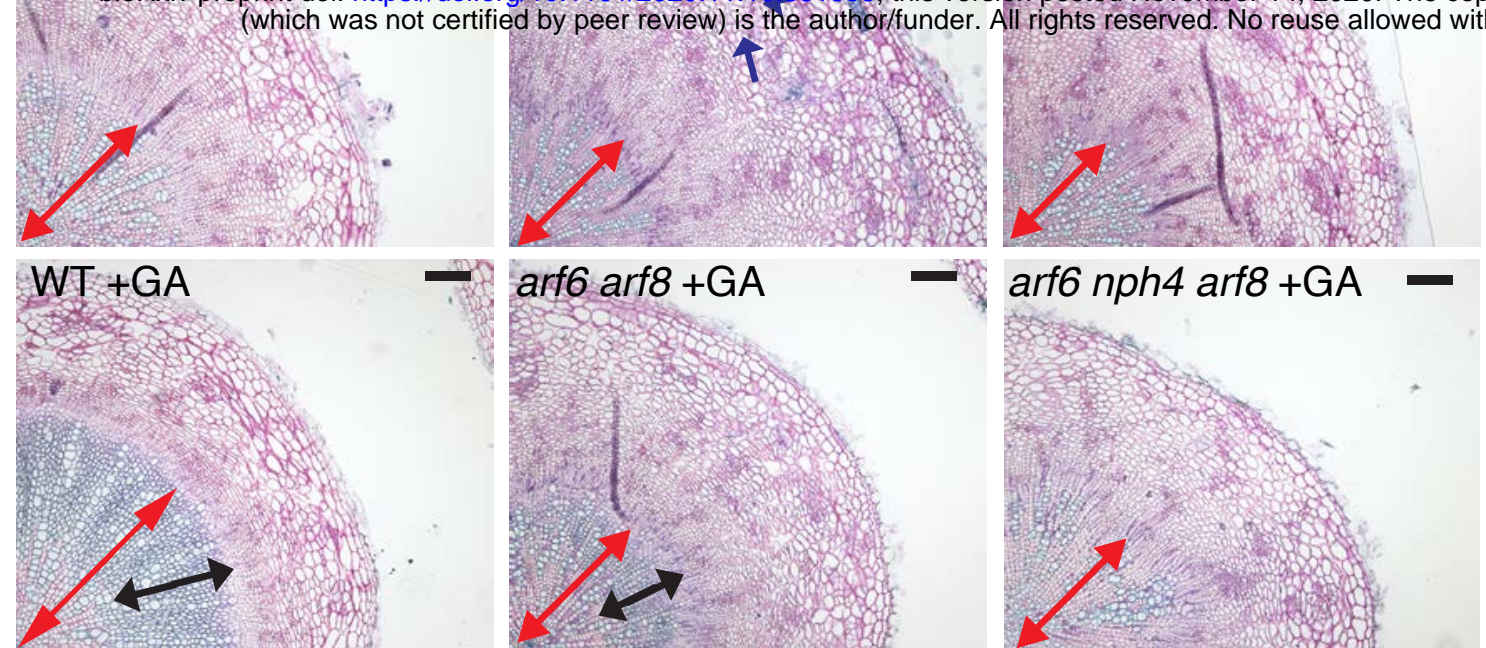

(b)

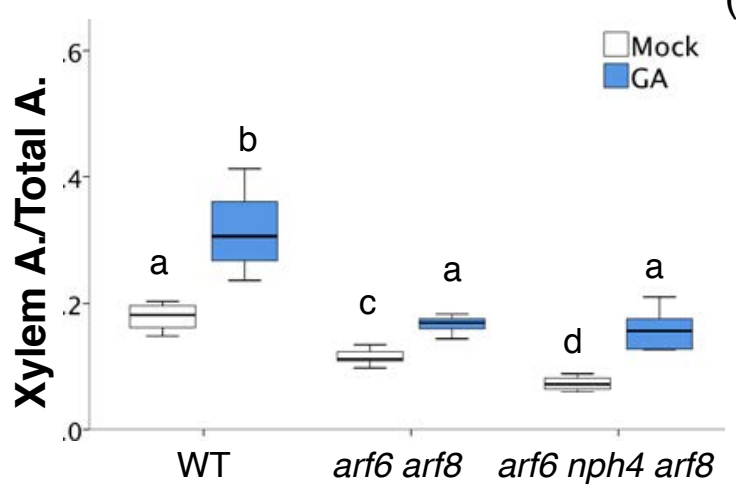

(c)
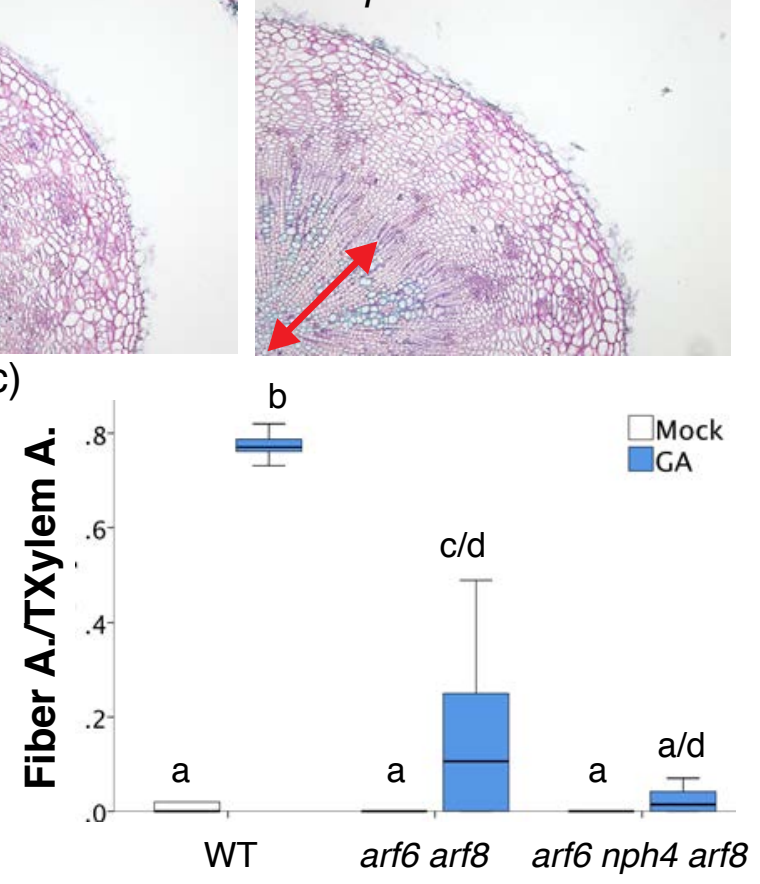

Fig.4 
(a)
WT
arf6 arf8
$b p$
arf6 arf8 bp

bioRxiv preprint doi: https://doi.org/10.1101/2020.11.13.381558; this version posted November 14,2020.The copyright (which was not certified by peer review) is the author/funder. All rights reserved. No reuse allowed without $p$

(b)

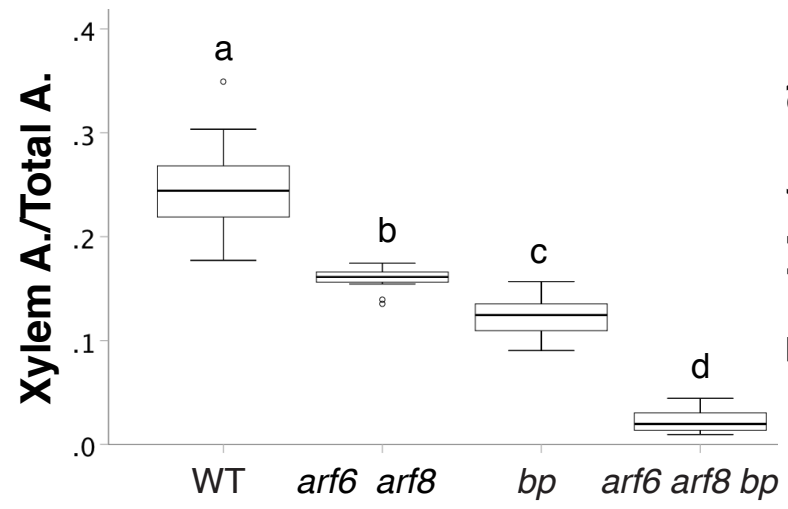

(c)

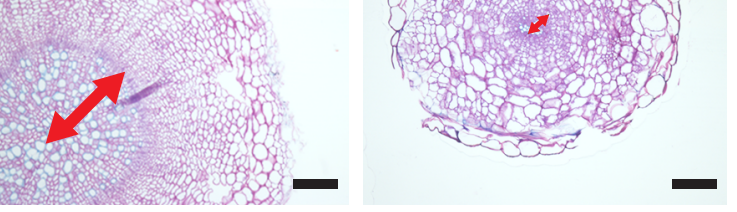

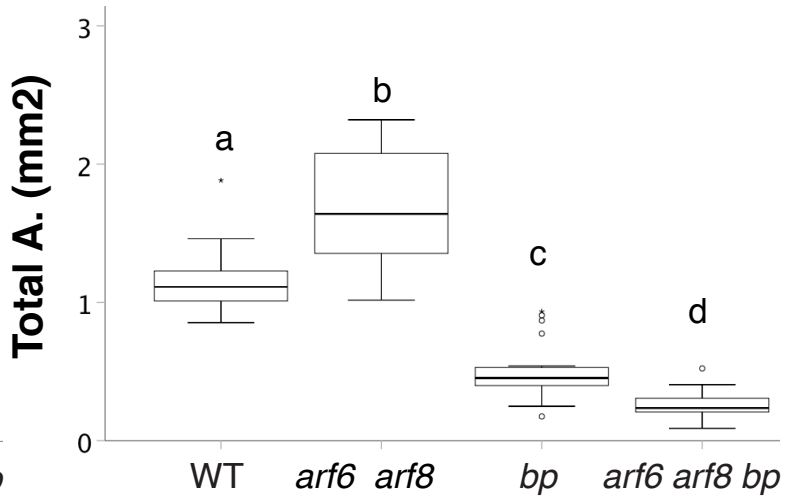

(d)
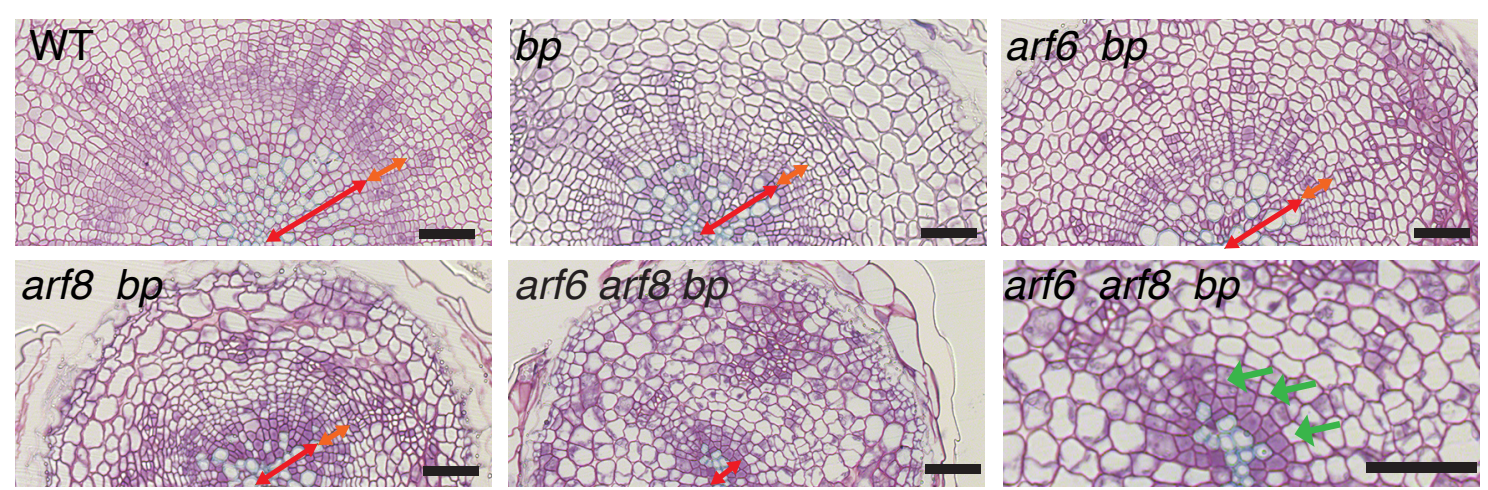

$(\mathrm{e})$

(f)

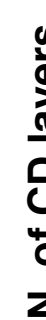

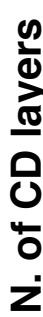
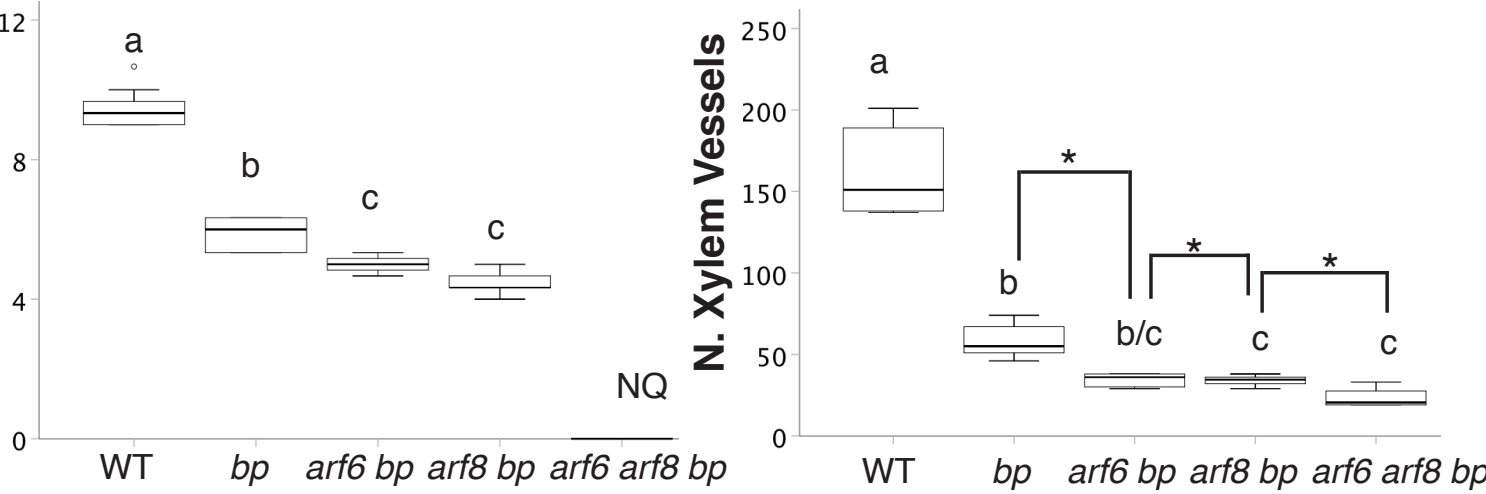

Fig.5 


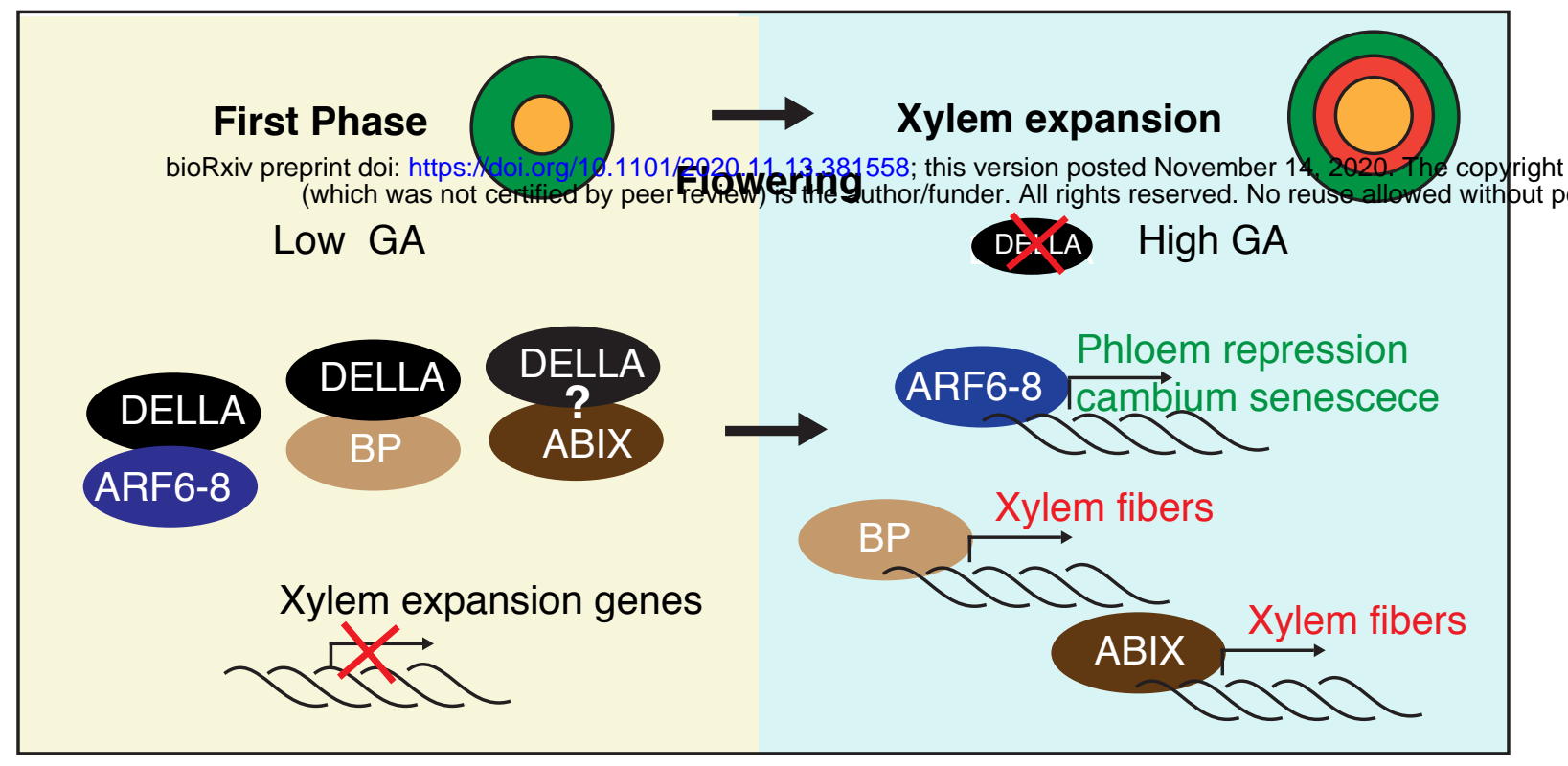

Fig.6 